\title{
Control of auxin-regulated root development by the Arabidopsis thaliana
}

\section{SHY2/IAA3 gene}

\author{
Qing Tian and Jason W. Reed* \\ Department of Biology, University of North Carolina at Chapel Hill, CB \#3280, Coker Hall, Chapel Hill, NC 27599-3280, USA \\ *Author for correspondence (e-mail: jreed@email.unc.edu) \\ Accepted 24 November 1998; published on WWW 20 January 1999
}

\section{SUMMARY}

The plant hormone auxin controls many aspects of development and acts in part by inducing expression of various genes. Arabidopsis thaliana semidominant shy 2 (short hypocotyl) mutations cause leaf formation in darkgrown plants, suggesting that SHY2 has an important role in regulating development. Here we show that the SHY2 gene encodes $I A A 3$, a previously known member of the $A u x / I A A$ family of auxin-induced genes. Dominant shy 2 mutations cause amino acid changes in domain II, conserved among all members of this family. We isolated loss-of-function shy 2 alleles including a putative null mutation. Gain-of-function and loss-of-function shy2 mutations affect auxin-dependent root growth, lateral root formation, and timing of gravitropism, indicating that SHY2/IAA3 regulates multiple auxin responses in roots. The phenotypes suggest that SHY2/IAA3 may activate some auxin responses and repress others. Models invoking tissue-specificity, feedback regulation, or control of auxin transport may explain these results.

Key words: auxin, SHY2, IAA3, Arabidopsis thaliana, root development

\section{INTRODUCTION}

Plant hormones are central endogenous regulators of many aspects of plant growth and development. Auxin, one of the most extensively studied hormones, regulates cell division, cell elongation, cell differentiation, and patterning (Sachs, 1991; Thimann, 1977). In the intact plant, auxin can inhibit root elongation, promote hypocotyl and stem elongation, mediate root and shoot gravitropism, promote lateral root and adventitious root formation, promote root hair formation, and maintain apical dominance (Estelle and Klee, 1994; Hobbie, 1998). A variety of studies suggest that auxin is also involved in patterning events such as vascular tissue differentiation and phyllotaxy (Sachs, 1991).

Genetic studies in Arabidopsis thaliana have yielded at least three classes of mutants with auxin-related phenotypes. One class of mutations affect genes involved in activation of the ubiquitin related protein Rub (such as axrl), or its putative downstream effectors tirl and sarl (Cernac et al., 1997; del Pozo et al., 1998; Ruegger et al., 1998). A second class of mutations affect auxin transport. For example, AUXI encodes an putative auxin uptake carrier and EIRl encodes a putative auxin efflux carrier (Bennett et al., 1996; Luschnig et al., 1998; Yamamoto and Yamamoto, 1998). Several other loci may also fall in this class, such as pinoid, pin-formed, rcn1, pis1, tir3 and lop1 (Bennett et al., 1995; Carland and McHale, 1996; Fujita and Syono, 1997; Garbers et al., 1996; Okada et al., 1991; Ruegger et al., 1997). A third class of mutations affect auxin-inducible genes or transcription factors that regulate such genes, such as axr3 and monopteros.

Several gene families induced rapidly and specifically by auxin have been identified in various plant species (Abel and Theologis, 1996). The Aux/IAA gene family has at least 20 members in Arabidopis thaliana (Abel et al., 1995; Kim et al., 1997). The kinetics of induction by auxin are different among these various genes, and some are induced faster than others. For example, induction of IAA3 and IAA6 mRNA begins within 5 minutes of auxin treatment and peaks after 10 minutes (Abel et al., 1995). Pea PS-IAA4 and PS-IAA6 proteins are localized to the nucleus and are very short-lived, having half-lives of 68 minutes, which suggests that the proteins play transient regulatory roles in response to auxin (Abel et al., 1994). The predicted proteins contain four conserved domains. Domains I, II and IV contain typical NLS motifs (Abel and Theologis, 1995). Domain III is predicted to form a $\beta \alpha \alpha$ structure, which is similar to the DNA-binding domain from certain prokaryotic repressors. However, there is no evidence that IAA proteins bind DNA directly. Domains III and IV are required for homoand hetero-dimerization of IAA proteins (Kim et al., 1997; Ulmasov et al., 1997b).

ARF (Auxin Response Factor) proteins are transcription factors that can bind to auxin response elements present in promoters of numerous auxin-regulated genes, including those of many IAA genes (Abel and Theologis, 1996; Guilfoyle et al., 1998a,b; Ulmasov et al., 1997a). There are at least 10 members of this family in Arabidopsis thaliana, and most of these (except for ARF3) have C-terminal domains homologous 
to domains III and IV of the IAA proteins (Guilfoyle et al., 1998b; Kim et al., 1997). ARF1 binds DNA through its Nterminal VP1-like motif, and its domain III- and IV-like Cterminal domain can interact with IAA proteins in the yeast two-hybrid system (Ulmasov et al., 1997b).

Mutants in several $I A A$ and $A R F$ genes have provided functional evidence that they are important in mediating vascular tissue differentiation, patterning and other auxin responses. Semidominant axr3 mutations cause increased apical dominance, reduced root elongation, increased adventitious root formation, auxin-resistant root growth, and upward curling of the leaf edges (Leyser et al., 1996). AXR3 has been cloned and shown to be IAA17, a member of $A u x / I A A$ gene family (Rouse et al., 1998). monopteros ( $\mathrm{mp}$ ) mutants are defective in auxin transport, vascular strand differentiation and apical-basal axis formation in early embryos (Przemeck et al., 1996). ettin mutants have defects in defining floral organ number and patterning in the gyneocium (Sessions et al., 1997). The MONOPTEROS and ETTIN genes encode ARF5 and $A R F 3$, respectively (Hardtke and Berleth, 1998; Sessions et al., 1997).

Semidominant shy2 mutations were identified in screens for suppressors of the long hypocotyl phenotype of phytochromedeficient hy 2 or phyB mutants (Kim et al., 1996; Reed et al., 1998). shy2-2 seedlings have short hypocotyls both in the dark and in the light, have curled-up leaves in the light, and flower early (Reed et al., 1998). shy2-2 mutants can also make leaves in the dark. shy2-3 mutants have less severe phenotypes, for example expanded cotyledons without extensive leaf formation in the dark (Reed et al., 1998). Similarly, the shy2-1 mutant, isolated as a suppressor of a hy 2 mutation, has curled-up leaves in the light, and can make true leaves, inflorescence stems and flowers in the dark (Kim et al., 1996, 1998). In addition, lightinduced $C A B$ and PSBA genes are expressed in dark-grown shy2-1 seedlings (Kim et al., 1998).

In this work, we report that shy 2 mutations fall in $I A A 3$, a member of the $A u x / I A A$ gene family. We have isolated loss-offunction alleles of $S H Y 2 / I A A 3$ and characterized phenotypes of both gain-of-function and loss-of-function shy 2 mutants. Our data indicate that SHY2/IAA3 plays a central role in auxin regulation of root growth, in gravitropism, and in lateral root formation.

\section{MATERIALS AND METHODS}

\section{Mapping}

We crossed shy2-2 (in Landsberg erecta) with Columbia wild type, and selected $\mathrm{F}_{2}$ plants of wild-type phenotype for analysis. Genomic DNA from individual $F_{2}$ plants was isolated and amplified with primers for various PCR-based CAPS markers including PVV4, NCCI, 0846A and IGF20G19 (Konieczny and Ausubel, 1993; Rouse et al., 1998), then assayed for Landsberg erecta and Columbia-specific polymorphisms.

\section{PCR sequencing}

We amplified the SHY2/IAA3 gene from shy2 mutants. The primers were 5'-AAA ACT GCA GAC ACT CAG CTT CCT CGA AGC-3' and 5'-CCG GGA TCC GGA GTA ATC CAA CCT CGA CC-3', and were designed based on the complete DNA sequence of the SHY2/IAA3 gene in GenBank from ecotype Columbia (PstI site and BamHI site are underlined). PCR reaction mixtures contained $100 \mathrm{ng}$ genomic DNA, $0.5 \mu \mathrm{M}$ of each primer, 0.5 unit of Taq polymerase, $0.2 \mathrm{mM}$ dNTP, $1.5 \mathrm{mM} \mathrm{MgCl}$, and reaction buffer $(20 \mathrm{mM}$ Tris- $\mathrm{HCl}$, $\mathrm{pH} 4.8,50 \mathrm{mM} \mathrm{KCl})$, and were incubated in a thermocycler for 34 cycles following hot start. Each cycle consisted of $94^{\circ} \mathrm{C}$ ( 45 seconds), $56^{\circ} \mathrm{C}$ ( 1 minute), $72^{\circ} \mathrm{C}$ ( 2 minutes) followed by 5 minutes at $72^{\circ} \mathrm{C}$. For shy2-2, the amplified genomic DNA fragments were cloned into pBluescript $\mathrm{SK}^{-}$(PstI/BamHI) by standard restriction enzyme digestion and ligation protocols and sequenced by standard methods. For shy2-3, shy2-21, shy2-22, shy2-23 and shy2-24, we amplified SHY2/IAA3 in 10 individual reactions and mixed them together, then purified the mixture by running through a Sephadex G50 column to remove primers and small nucleotides. The purified PCR product was sequenced directly by standard methods.

\section{Generation of transgenic plants}

A $3.5 \mathrm{~kb}$ fragment of genomic DNA including the SHY2/IAA3 coding sequence and $2.2 \mathrm{~kb}$ of upstream DNA was amplified from shy2-2 DNA using the primers $5^{\prime}$-AGT GCT ATA ATC AAC CAG CG-3' and 5'-CCG GGA TCC GGA GTA ATC CAA CCT CGA CC-3' (underlined is the BamHI site) and cloned into pPZP211 vector (PstI/EcoRI) (Hajdukiewicz et al., 1994). (The methods for PCR amplification and cloning were as described above except that the annealing temperature was $60^{\circ} \mathrm{C}$ intead of $56^{\circ} \mathrm{C}$.) The plasmid was introduced into Agrobacterium strain GV3101 (Koncz and Schell, 1986) by electroporation. Wild-type plants (Landsberg erecta) were inoculated with this strain by vacuum infiltration (Bechtold et al., 1993). Transformed seedlings were identified by selection on 50 $\mu \mathrm{g} / \mathrm{ml}$ kanamycin.

\section{Mutagenesis and suppressor screen}

We incubated 1800 shy2-2 $\mathrm{M}_{1}$ seeds overnight in $0.07 \%$ ethyl methane sulfonate (EMS) and 1800 seeds in $0.2 \%$ ethyl methane sulfonate, and then rinsed them several times with water. We collected $\mathrm{M}_{2}$ progeny in 10 batches from $350 \mathrm{M}_{1}$ plants per batch. We screened $70,000 \mathrm{M}_{2}$ seedlings on MS/SUC/agar plates under red light for those with a long hypocotyl. To detect the starting shy2-2 mutation, we PCR amplified the SHY2/IAA3 gene from genomic DNA, and assayed for the presence (wild-type allele) or absence (shy2-2 mutant allele) of a HaeIII site. Mutants from different batches were assumed to be independent. PCR products of the SHY2/IAA3 gene from the intragenic suppressor alleles were sequenced as described above.

\section{RT-PCR}

RNA was isolated as described by Chory et al. (1991). Complementary DNA synthesis was performed in a reaction consisting of $13.3 \mathrm{U} / \mu 1$ reverse transcriptase, $1 \times$ reverse transcription buffer (50 mM Tris- $\mathrm{HCl}, 8 \mathrm{mM} \mathrm{MgCl} 2,10 \mathrm{mM}$ dithiothreitol), 0.5 $\mathrm{mM}$ dNTP mix, $1 \mathrm{U} / \mu \mathrm{l}$ RNAse inhibitor, and $1.5 \mu \mathrm{M}$ oligo(dT) primer. RNA $(10 \mu \mathrm{g})$ in $19 \mu \mathrm{l}$ was denatured at $68^{\circ} \mathrm{C}$ for 3 minutes, then the reaction buffer was added and the mixture incubated at $42^{\circ} \mathrm{C}$ for 1 hour. $3 \mu \mathrm{l}$ of the products were then used for PCR after denaturation for 10 minutes at $68^{\circ} \mathrm{C}$. PCR methods were as described above. The primers were $5^{\prime}$-CAG AGC TGA GGC TGG GAT TAC$3^{\prime}$ and $5^{\prime}$-CGG GAT CCC GTA CAC CAC AGC CTA AAC-3' (the BamHI site is underlined). We found that these primers amplified a fragment of IAA4 cDNA as well as the $S H Y 2 / I A A 3$ cDNA. Therefore, the PCR products were cloned into pBluescript $\mathrm{SK}^{-}$(Stratagene, La Jolla, CA), and a SHY2/IAA3-specific clone was identified and sequenced.

\section{Seedling growth experiments}

Seeds were surface sterilized and plated on MS/agar plates (1× Murashige and Skoog salts, $0.8 \%$ phytagar [Gibco BRL], $1 \times$ Gamborg's B5 vitamin mix) with or without $2 \%$ sucrose, and stored at $4^{\circ} \mathrm{C}$ overnight to induce germination. The plates were grown at $23^{\circ} \mathrm{C}$ under a mixture of fluorescent and incandescent bulbs at an intensity of $100-200 \mu$ moles $/ \mathrm{m}^{2}$ second. For dark experiments, seeds 
were induced to germinate in the light for 12 hours after cold treatment, and then transferred to total darkness.

For hypocotyl and root growth rate experiments, seedlings were grown on vertically oriented MS/SUC/agar plates under white light or in the dark. Digital images of the plates were taken daily after germination with a CCD camera and the hypocotyl and root lengths were measured using image analysis software (NIH image, Bethesda, $\mathrm{MD})$. The root growth rates of all genotypes varied quantitatively from experiment to experiment, presumably as a result of variable light, humidity, temperature, or other aspects of the growth conditions, and representative root growth experiments are shown. For the dark experiment, one plate was used for each time point. For measurement of cotyledon sizes, seedlings were grown on horizontally oriented plates in white light for 10 days, and the cotyledons were excised and flattened under a cover slip. The cotyledon sizes were then measured using a microscope equipped with a camera lucida.

\section{Lateral and adventitious root formation}

For measurement of lateral root formation, seedlings were grown on vertically oriented MS/SUC/agar plates under white light for 3 weeks, and the number of lateral roots was counted under a dissecting microscope. For measurement of adventitious root formation, the seeds were surface sterilized and plated on MS/SUC/agar plates, stored at $4^{\circ} \mathrm{C}$ for 3 days, and grown horizontally under white light for 1 day at $23^{\circ} \mathrm{C}$. Then the plates were placed vertically in the dark for 5 days, moved to white light again, and set horizontally for 18 days. Adventitious roots were counted under a dissecting microscope.

\section{Gravitropism}

Seedlings for the gravitropic angle experiment were grown under different fluence rates of red light on vertically oriented plates as described previously (Reed et al., 1998). After 5 days growth, images were taken with a CCD camera, and angles between the growing direction of hypocotyls and the opposite direction of gravity (hypocotyl gravitropism), and angles between the growing direction of roots and the direction of gravity (root gravitropism) were measured using image analysis software (NIH image). For the gravitropic curvature experiment, seedlings were grown on vertically oriented MS/SUC/agar plates in the dark for 48 hours and then were rotated through $90^{\circ}$ in the darkness. For measurement of hypocotyl and root gravitropic curvature, we measured growth angles only of seedlings for which the orientation of hypocotyls and roots before re-orientation was less than $20^{\circ}$ from vertical. The angles between the opposite direction of gravity or the direction of gravity after re-orientation and the growing direction of hypocotyls or roots at each time was measured as the curvature. One plate was used for each time point.

\section{Wavy root examination}

Seedlings were grown on MS/SUC/1.5\% agar plates set in a vertical position for 3 days. Then the plate was tilted to $45^{\circ}$ and the seedlings were grown for 3 days and photographed.

\section{Root growth response to exogenous hormones}

Seedlings were grown on vertically oriented MS/SUC/agar plates for 5 days under white light at $23^{\circ} \mathrm{C}$ to allow the roots to grow along the surface. Then 20 seedlings per treatment were transferred to new plates supplemented with various concentrations of hormones (IAA, 2,4-D, $\mathrm{BA}, \mathrm{ABA}$, or NPA). The positions of root tips of all seedlings were marked. After 3 days, the amount of new root growth was measured.

\section{RESULTS}

\section{Cloning of SHY2}

Previous work showed that SHY2 maps to chromosome 1 between markers PVV4 and nga63. We mapped the gene more precisely using two polymorphic CAPS markers in this region developed by Rouse et al. (1998). These data established that SHY2 is north of 0846A, close to IGF20G19 (Fig. 1). A sequenced BAC clone, IGF19P19, extends $16 \mathrm{~kb}$ into this region and contains two adjacent $A u x / I A A$ genes, IAA3 and $I A A 17$ (Fig. 1). AXR3 also maps to this region and it has been demonstrated to be IAA17 (Rouse et al., 1998).

shy 2 and axr3 mutants share several phenotypes, such as short hypocotyls, curled-up leaves and leaf formation in darkness. However, unlike shy 2 mutants, axr3 mutants have few shoot branches and highly agravitropic roots that lack root hairs (Leyser et al., 1996). These observations suggested that shy 2 mutations might affect the other $A u x / I A A$ gene family member in this region, IAA3. We demonstrated this by sequencing the IAA3 gene from shy 2 mutants. shy2-2 has a single nucleotide mutation predicted to change the proline at amino acid position 69 to serine (Fig. 2). shy2-3 has a single nucleotide change predicted to change the glycine at amino acid position 67 to glutamine (Fig. 2). Both of these mutations affect amino acid residues in domain II, a highly conserved domain among the Aux/IAA proteins. Semidominant axr3-1 and axr3-3 mutations affect amino acids in the corresponding domain of $I A A 17$, suggesting that this domain has a similar regulatory function in both proteins (Rouse et al., 1998).

We transformed the SHY2/IAA3 gene together with about 2.2 $\mathrm{kb}$ of DNA upstream of the open reading frame from shy2-2 mutant genomic DNA into wild-type (Landsberg erecta) plants. 14 out of $15 \mathrm{~T} 1$ transformants resembled shy2-2 plants in having upcurled leaves and short hypocotyls (Fig. 3P,Q). These data show that the point mutation in shy2-2 is sufficient to confer the characteristic shy 2 phenotype, and suggest that $2.2 \mathrm{~kb}$ of upstream sequence is sufficient to direct normal expression. Together with the finding that shy2-2 and shy2-3 are semidominant missense mutations, this result suggests that these mutations cause gain of function.

\section{Loss-of-function alleles of SHY2/IAA3}

We isolated four potential loss-of-function alleles of SHY2/IAA3 by mutagenizing shy2-2 with EMS and screening for seedlings with long hypocotyls in red light. $\mathrm{F}_{1}$ plants from backcrosses of each suppressor to shy2-2 plants resembled heterozygous shy2-2 plants, indicating that the new mutations are recessive to shy2-2. $\mathrm{F}_{1}$ plants from crosses to wild type

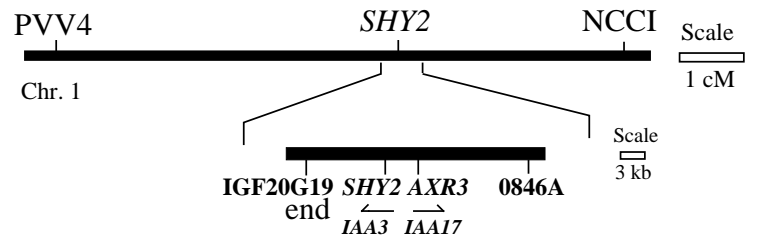

Recombinants

$\begin{array}{cccc}13 / 236 & 0 / 236 & 0 / 236 & 0 / 236 \\ 0 / 236 & 0 / 236 & 1 / 236 & 9 / 236\end{array}$

Fig. 1. Cloning the $S H Y 2$ gene. The position of the $S H Y 2$ locus is shown relative to chromosome one CAPS markers PVV4, NCCI, 0846A and IGF20G19 end. Numbers beneath the line indicate frequencies of recombinants between $S H Y 2$ and each marker. The position and transcription direction (arrows) of AXR3/IAAI7 and SHY2/IAA3 are also shown. 
Fig. 2. Sequences of shy2 mutations. Three exons (boxes) and two introns (lines) of the SHY2/IAA3 gene are shown. Black boxes indicate the open reading frame. Positions of shy2 mutations and corresponding amino acid changes are indicated. shy2-22 retains the shy2-2 mutation and has aberrant splicing causing a reading frame shift in the third exon. The altered amino acid sequence is indicated. shy2-21 is identical to shy2-22, and

shy2-23 is identical to shy2-24.

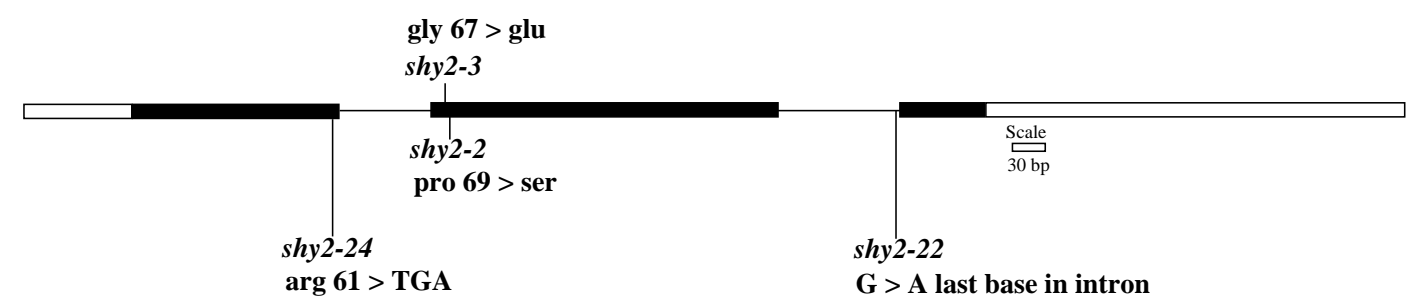

appeared similar to wild type, as did all of the $\mathrm{F}_{2}$ plants from self-fertilization of the $F_{1}$ plants. These data showed that the suppressor mutations are closely linked to the shy2-2 mutation. We sequenced the $S H Y 2 / I A A 3$ gene from the four tall mutants, and found that all four retained the shy2-2 mutation and also had an additional point mutation at another position in the gene. Accordingly, we refer to these alleles as shy2-21, shy222, shy2-23 and shy2-24.

shy2-21 and shy2-22 have identical mutations that change the consensus $3^{\prime}$ splice site $\mathrm{A} \underline{\mathrm{G}}$ of the second intron to $\mathrm{A} \underline{\mathrm{A}}$ (Fig. 2 ). We cloned and sequenced a RT-PCR product of the SHY2/IAA3 gene from shy2-22 seedlings and found that the mutation causes aberrant splicing. Splicing of the second intron uses the mutated $\underline{\mathrm{A}}$ in the last position of the second intron and the $G$ in the first position of the third exon as an alternative 'consensus' AG splicing site. This altered splicing shifts the reading frame of the third exon, thereby replacing the last 24 amino acids at the C-terminus (including half of conserved domain IV) with 8 amino acids (Fig. 2).

shy2-23 and shy2-24 have identical mutations that introduce a stop codon at amino acid position 61,8 amino acids upstream of the codon mutated in shy2-2 (Fig. 2). Since the shy2-21, shy2-22, shy2-23 and shy2-24 alleles each have mutations that truncate the protein (all four alleles) and/or replace a domain (shy2-21 and shy2-22), they are probably loss-of-function alleles. As the shy2-23 and shy2-24 mutations truncate the protein by two thirds and confer more extreme phenotypes than the shy2-21 and shy2-22 mutations (see below), shy2-23 and shy2-24 may be null alleles. shy2-21 and shy2-22 are more complex, in that they appear to have a partial loss-of-function mutation and a gain-of-function mutation within the same gene.

\section{Shoot morphology of shy2 mutants}

Whereas gain-of-function shy2 mutations caused dramatic shoot phenotypes, loss-of-function mutations had more subtle effects. Light-grown shy2-2 and shy2-3 seedlings had larger cotyledons and shorter hypocotyls than wild type seedlings after 10 days of growth, and their cotyledons curled up (Table 1; Fig. 3B; Reed et al., 1998). shy2-2 and shy2-3 adult plants were extremely dwarfed with curled-up leaves (Fig. 3F,G). As described previously for hypocotyl elongation, the adult phenotype conferred by shy2-2 was semidominant (Fig. $3 \mathrm{H}$ ). In contrast, shy2-22 and shy2-24 seedlings did not have strong phenotypes in the shoot. The hypocotyls of shy2-22 and shy224 seedlings grew slightly faster than wild type at early times, but slowed resulting in similar final hypocotyl lengths to wildtype seedlings (Fig. 3A,C,D, 4A). Cotyledons of shy2-22, but not shy2-24, seedlings were slightly larger than wild-type cotyledons (Table 1). In contrast to the upcurled cotyledons and leaves of shy2-2 and shy2-3 plants, those of shy2-22 and shy224 plants appeared normal (Fig. 3C,D) and adult shy2-22 and shy2-24 plants appeared similar to wild-type plants (Fig. 3I,J).

One-week old dark-grown wild-type seedlings elongate their hypocotyls, have unexpanded cotyledons, retain an apical hook, and fail to develop leaves (Fig. 3K). In contrast, shy2-2 seedlings had short hypocotyls, expanded cotyledons, opened apical hooks, and true leaf primordia (Fig. 3L; Reed et al.,

Table 1. Measurement of shy 2 mutants

\begin{tabular}{|c|c|c|c|c|c|}
\hline & $S H Y 2$ & shy2-2 & shy2-3 & shy2-22 & shy2-24 \\
\hline Hypocotyl length (mm) & $2.9 \pm 0.8$ & $1.3 \pm 0.3 * * *$ & $1.7 \pm 0.5 * * *$ & $2.9 \pm 0.5 \dagger$ & $2.8 \pm 0.5 \dagger$ \\
\hline Root length (mm) & $25.6 \pm 0.9$ & $16.5 \pm 3.7 * * *$ & $20.7 \pm 2.6 * * *$ & $16.2 \pm 6.1 * * *$ & $11.5 \pm 4.2 * * *$ \\
\hline Cotyledon size $\left(\mathrm{mm}^{2}\right)$ & $5.6 \pm 0.8$ & $6.7 \pm 0.4 * * *$ & $7.6 \pm 0.8 * * *$ & $7.1 \pm 0.9 * * *$ & $6.0 \pm 0.4 \dagger$ \\
\hline $\begin{array}{l}\% \text { of } 21 \text {-day dark-grown seedlings having } \\
\text { visible leaf primodia }\end{array}$ & 7 & 100 & 24 & 9 & 22 \\
\hline Lateral roots/seedling & $9.1 \pm 4.6$ & $2.1 \pm 2.1 * * *$ & ND & $14.7 \pm 7.4 * *$ & $16.4 \pm 7.9 *$ \\
\hline Adventitious roots/seedling & $2.3 \pm 1.7$ & $0.1 \pm 0.4 * * *$ & $0.1 \pm 0.2 * * *$ & $2.3 \pm 1.5 \dagger$ & $2.8 \pm 2.0 \dagger$ \\
\hline
\end{tabular}


1998). shy2-3 mutants had phenotypes similar to but less severe than those of shy2-2 mutants (Fig. 3M; Reed et al., 1998). In contrast, shy2-22 and shy2-24 mutants appeared almost identical to wild type (Fig. 3N,O). shy2-22 and shy224 hypocotyl growth slowed slightly after growing similarly to wild type at early times (Fig. 4B). After 3 weeks of dark growth, all shy2-2 mutant seedlings formed visible leaf primordia, whereas very few wild type, shy2-22, or shy2-24 seedlings did (Table 1).

\section{Root morphology of shy2 mutants}

Both gain-of-function and loss-of-function shy2 mutations affected root growth. When grown in the light, all shy2 mutant seedlings had significantly shorter roots than wild-type seedlings (Table 1). The short root phenotype was most severe in the shy2-24 mutant, and was reflected in reduced root growth rates compared to wild-type seedlings (Fig. 4C). When grown in the dark, all shy 2 seedlings had average root growth rates similar to those of wild-type seedlings (Fig. 4D).

The shy 2 mutations also affected root branching. When grown on vertically oriented plates in continuous white light for 3 weeks, shy2-2 mutants made very few lateral roots, whereas the shy2-22 and shy2-24 mutants formed more and much longer lateral roots than wild-type seedlings (Table 1, Fig. 5). When grown under conditions that promote adventitious root formation in the hypocotyl, shy2-2 and shy2-3 seedlings each made fewer adventitious roots than wild type (Table 1). Although they made excess lateral roots, shy2-22 and shy2-24 plants each made similar numbers of adventitious roots to wild type (Table 1).

\section{Auxin response phenotypes}

The discovery that SHY2/IAA3 encodes an auxin-regulated gene suggests that it might play a role in auxin responses. Moreover, alterations in lateral root number and in distribution of seedling growth are consistent with changes in auxin physiology. To investigate directly the effects of SHY2/IAA3 on auxin responses, we tested
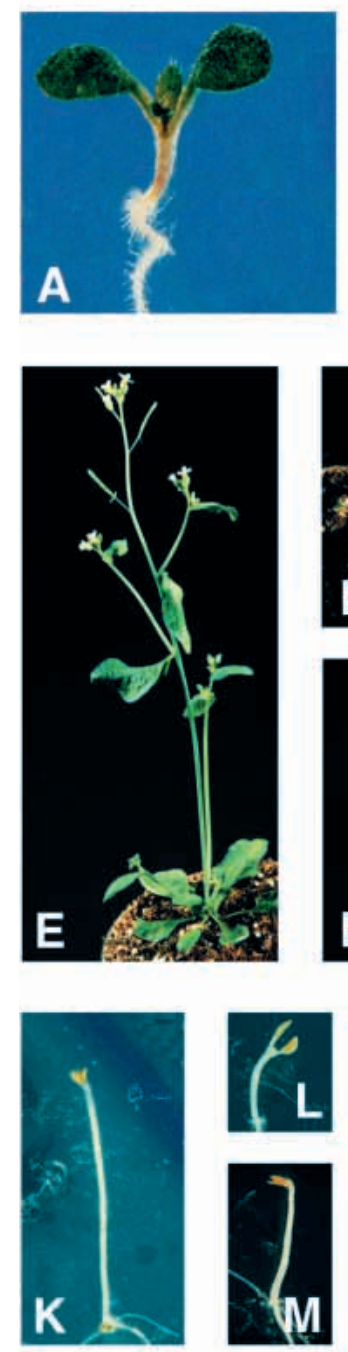

whether shy2 mutant seedlings could respond to gravity, a known auxin-regulated response; and we measured the effect of exogenous auxin on root growth.

\section{Root and hypocotyl gravitropism}

When grown in the dark, hypocotyls of wild-type seedlings grew upward, and roots grew down. Hypocotyls and roots of shy2-2 mutants grew in more random directions (Fig. 6). In contrast, shy2-22 and shy2-24 mutants grew vertically as wild type seedlings did (Fig. 6). Light perceived by phytochromes modulates hypocotyl gravitropism (Gaiser and Lomax, 1993; Liscum and Hangarter, 1993; Robson and Smith, 1996). To assess whether shy2 mutations altered this response, we measured the angle (relative to vertical) of hypocotyl and root growth under different fluence rates of red light. Wild-type seedlings responded with randomization of the growth direction of hypocotyls. shy2-2 seedlings did not respond to red light, whereas shy2-22 and shy2-24 seedlings responded similarly to wild type (Fig. 6). These data indicate that
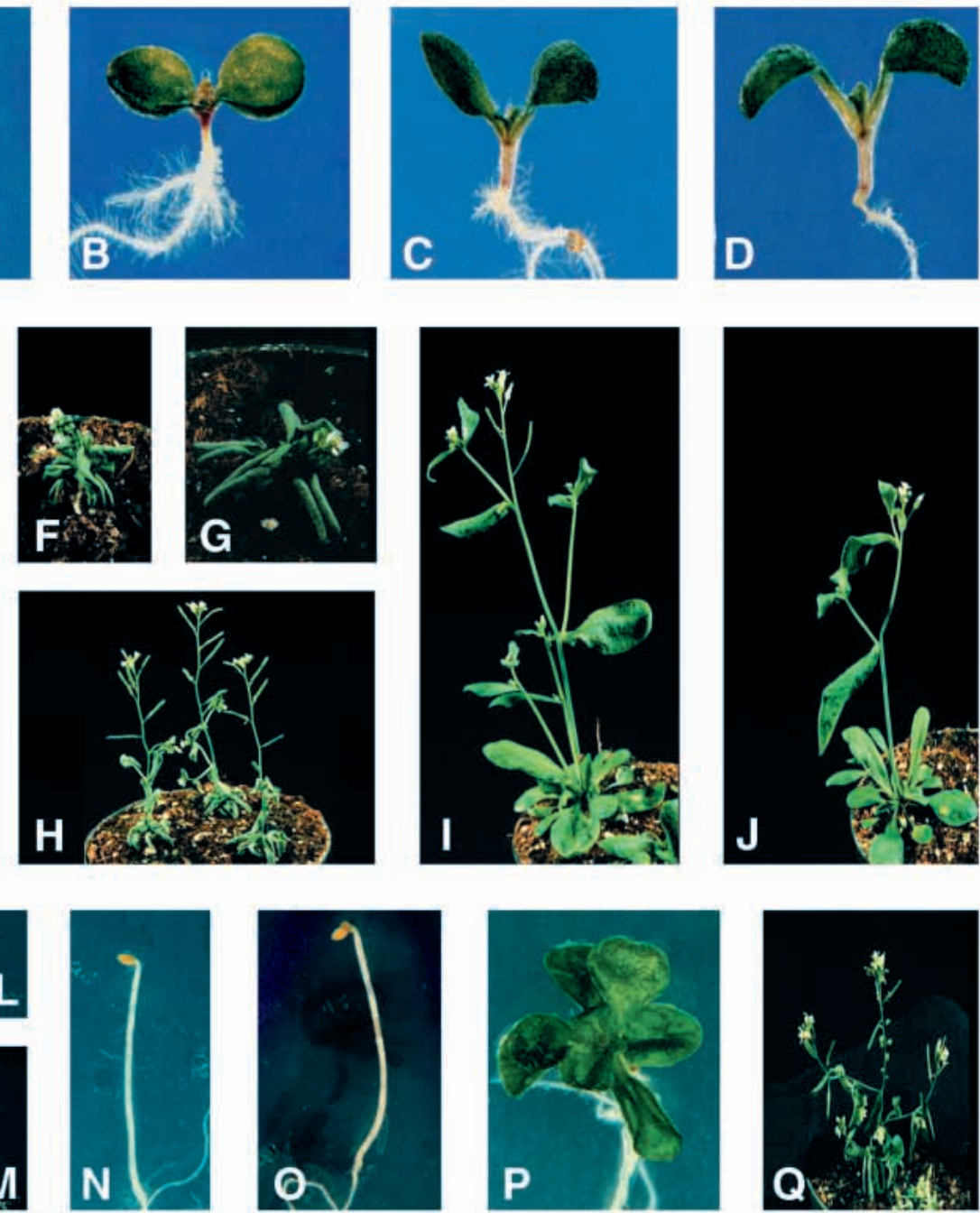

Fig. 3. Phenotypes of shy2 mutants. Seedlings were grown on MS/SUC plates in white light for 7 days (A$\mathrm{D})$, in white light for 25 days (E-J), or in the dark for 7 days (K-O). Genotypes are (A,E,K) Landsberg erecta (wild-type parent), (B,F,L) shy2-2, (G,M) shy2-3, (H) SHY2/shy2-2 heterozygous plants, (C,I,N) shy2-22, (D,J,O) shy2-24. shy2-21 and shy2-23 seedlings are similar to shy2-22 and shy2-24 seedlings, respectively, and are not shown. (P,Q) 14-day-old and 35-day-old Landsberg erecta plants carrying a shy2-2 transgene. 
gravitropic sensing is largely intact in shy 2 loss-of-function mutants.

However, an inductive experiment uncovered altered gravitropic response in shy 2 mutant seedlings. We re-oriented dark-grown seedlings by $90^{\circ}$ and then measured the kinetics of hypocotyl and root tip curvature. Hypocotyls and roots of shy22 and shy2-22 seedlings had a slower response to gravity than those of wild-type seedlings (Fig. 7A,B), although both hypocotyls and roots eventually re-oriented vertically (by 48 hours, data not shown). This result shows that although shy22 seedlings initially grow agravitropically, they retain a correctly oriented response to gravity stimulus. The similarity of the shy2-22 response to that of shy2-2 suggests that shy222 retains some of the gain-of-function activity conferred by the shy2-2 mutation. The putative null mutant shy2-24 had a contrasting phenotype. Hypocotyls of shy2-24 seedlings responded similarly to wild-type hypocotyls (Fig. 7A). However, the roots of shy2-24 seedlings re-oriented more quickly than wild-type roots (Fig. 7B). These results indicate that SHY2/IAA3 slows root curvature in response to gravity.

\section{Wavy root pattern of shy2 mutants}

These altered root curvature responses were reflected in altered wavy root growth on inclined $1.5 \%$ phytagar plates. Wavy root growth is thought to result either from a gravitropismdependent thigmotropic obstacleescaping response (Okada and Shimura, 1994), or, in an alternative model, from the combinational effects of circumnutation and gravitropism of the growing root tip (Simmons et al., 1995). As shown in Fig. 8, shy2-2 showed less wavy growth than wild type, similar to previously reported wav1-1 and hy5 mutants (Okada and Shimura, 1990; Oyama et al., 1997). Conversely, shy2-22 showed increased wavy growth, with each curve encompassing a larger angle than curves in wild-type roots, similar to wav2-1 mutants (Okada and Shimura, 1990). shy2-24 (and in some cases shy2-22) roots showed an even more pronounced phenotype. They formed less frequent but larger curves which often looped through more than a complete circle. These results confirm that SHY2/IAA3 affects the extent and/or timing of gravity response.

\section{Hormone sensitivity of root growth}

We tested whether the short roots of shy 2 mutants might arise from altered auxin response by measuring root growth in the presence of exogenous auxin. Both shy2-2 and shy2-3 are slightly less sensitive than wild type to

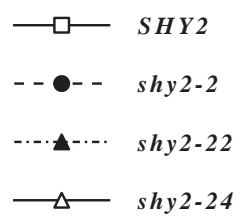

the natural auxin indole-3-acetic acid (IAA), by about 2-fold (Fig. 9A). This contrasts with axr3-1, which has a much more substantial auxin-resistance in this assay (about 500-fold; Leyser et al., 1996). The shy2-22 and shy2-24 mutants behaved unusually in this assay. The short root phenotype of shy2-22 and shy2-24 mutants was rescued by low concentrations of exogenous IAA $\left(1-3 \times 10^{-8} \mathrm{M}\right)$. Higher concentrations $\left(>10^{-7}\right.$ M) inhibited root growth, as for wild type and shy2-2 roots (Fig. 9B). The mutants had qualitatively similar responses to the synthetic auxin 2,4-D (data not shown). These striking results confirm that shy2 mutants have an altered auxin response.

To assess whether shy 2 affects auxin responses specifically, we tested root growth responses to other hormones. shy2-2 mutant seedlings were slightly resistant to abscisic acid (ABA; Fig. 9C), the cytokinin benzyladenine (BA; Fig. 9D), the auxin transport inhibitor naphthylphthalamic acid (NPA; Fig. 9E), and the ethylene precursor 1-aminocyclopropane-1-carboxylic acid (ACC, data not shown). Like IAA, low concentrations of BA $\left(<10^{-8} \mathrm{M}\right)$ (Fig. 9D) and NPA $\left(<10^{-7} \mathrm{M}\right)$ (Fig. 9E), but not ABA (Fig. 9C) stimulated root growth of shy2-22 and shy2-24
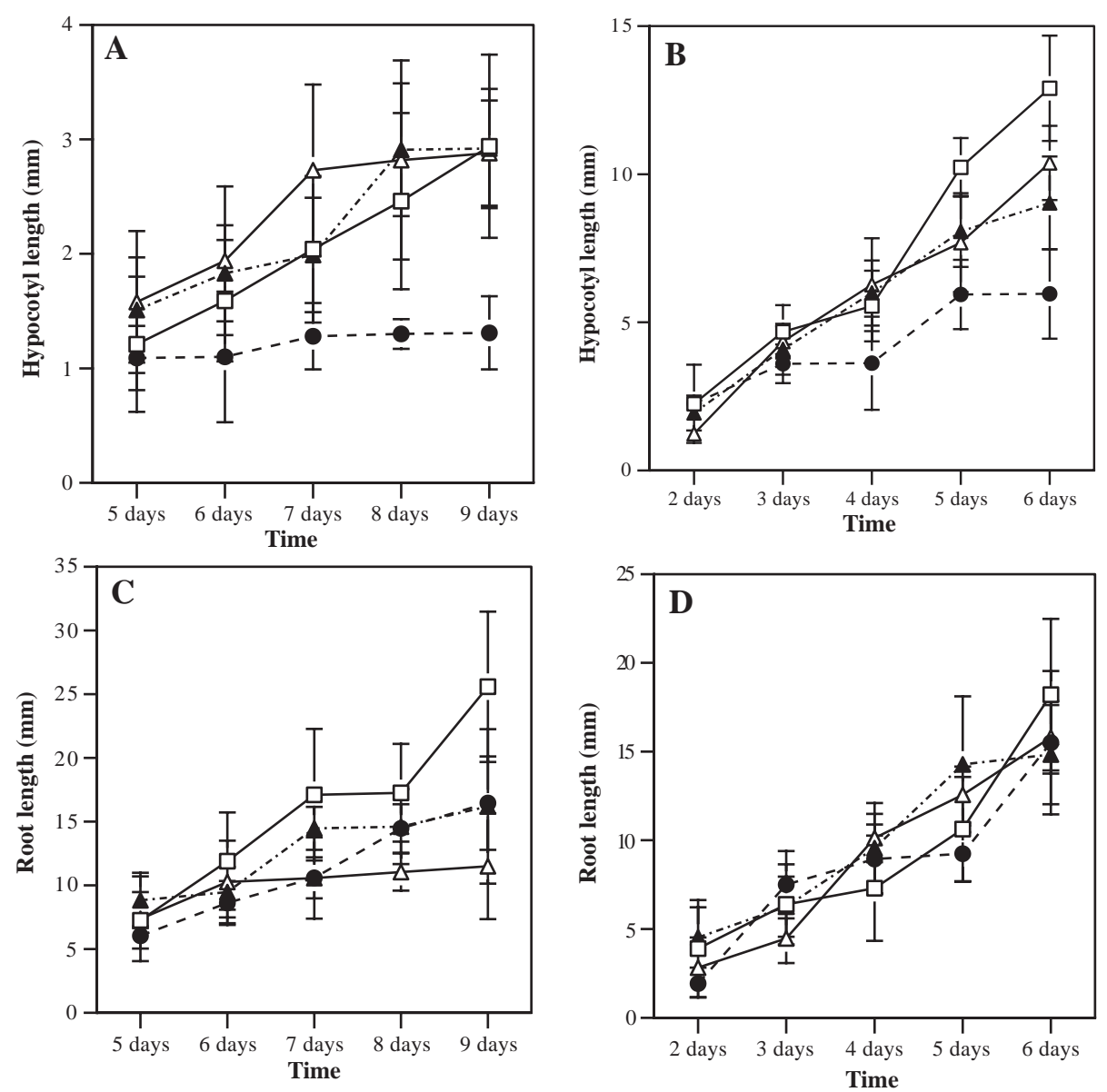

Fig. 4. Time course of hypocotyl and root elongation. (A,B) Hypocotyl, $(C, D)$ root. Seedlings were grown vertically in white light $(A, C)$ or dark (B,D) on MS/SUC plates. Hypocotyl and root lengths were measured daily. Error bars indicate standard deviations. At least 20 seedlings were measured for each point. Repetitions of the experiment shown in A revealed that shy2-24 hypocotyls consistently grew faster than wild-type seedling hypocotyls (data not shown). 


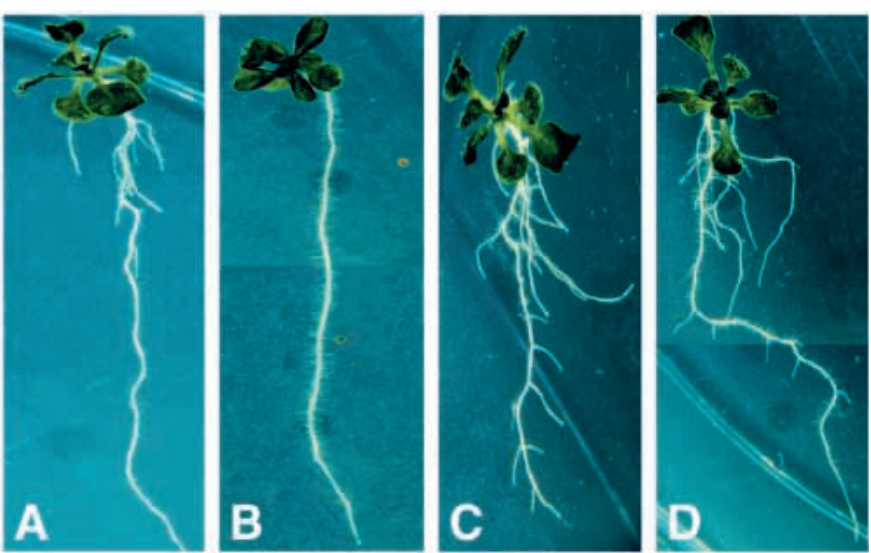

Fig. 5. Lateral root formation. (A) Wild type (Landsberg erecta), (B) shy2-2, (C) shy2-22, (D) shy2-24. Seedlings were grown on vertically oriented plates in white light for 21 days.

mutants. Other auxin-resistant mutants are also resistant to auxin, cytokinin, ABA, and/or auxin transport inhibitor (Hobbie and Estelle, 1995; Leyser et al., 1996; Pickett et al., 1990; Ruegger et al., 1998; Wilson et al., 1990). Our results reinforce the notion that there are significant functional connections among different hormone signalling components.

\section{DISCUSSION}

The availability of both gain-of-function and loss-of-function shy 2 alleles allows a complementary analysis of functions for which SHY2/IAA3 is sufficient and/or necessary. The gain-offunction and loss-of-function mutations affect several root phenotypes in opposing senses and reveal that SHY2/IAA3 regulates root system development. Thus, gain-of-function mutations cause reduced lateral root formation, decreased wavy root curvature and slow root re-orientation; whereas lossof-function mutations cause increased lateral root formation, increased wavy root curvature and accelerated root reorientation. Auxin is known to stimulate lateral root formation, and to be required for gravitropism. Therefore, these phenotypes are consistent with the interpretation that SHY2/IAA3 is a negative regulator of auxin responses.

Exogenous auxin rescued root growth of loss-of-function mutants suggesting that the morphological phenotypes of the mutants arise from altered response to auxin. However, these data are most consistent with SHY2/IAA3 being a positive regulator of auxin response, rather than a negative regulator as the morphological phenotypes suggest. Thus, loss-of-function mutants may have reduced response to auxin, thereby perceive and respond as if to a lower concentration of auxin than is actually present, and obtain growth stimulation at concentrations of exogenous auxin that normally inhibit wildtype root growth. Consistent with this interpretation, other workers have reported that very low concentrations $\left(10^{-11}\right.$ $10^{-10} \mathrm{M}$ ) of exogenous IAA can stimulate root growth, and higher concentrations of IAA inhibit root growth (Evans et al., 1994). Gain-of-function shy 2 mutants also had shorter roots than wild type, but rather than stimulating root growth as in loss-of-function mutants, exogenous auxin inhibited root growth, at slightly higher concentrations than required for wild-type. This slight auxin resistance may indicate that gainof-function mutant roots have a higher constitutive 'response' than wild type to endogenous auxin and therefore require a higher level of exogenous auxin to show further inhibition of root growth.

Several possible models may reconcile our inference that SHY2/IAA3 is both a positive and negative regulator of auxin responses. One possible interpretation is that SHY2/IAA3 acts differentially in different tissues. For example, SHY2/IAA3 may promote cell enlargement in cotyledons, but inhibit cell enlargement in hypocotyls and roots; and it may promote cell divisions in shoot and root apical meristems leading to leaf formation and primary root elongation, but inhibit pericycle cell divisions thereby preventing lateral root formation. Such tissue-specific effects might arise if SHY2/IAA3 dimerizes with alternate Aux/IAA or ARF proteins present in different
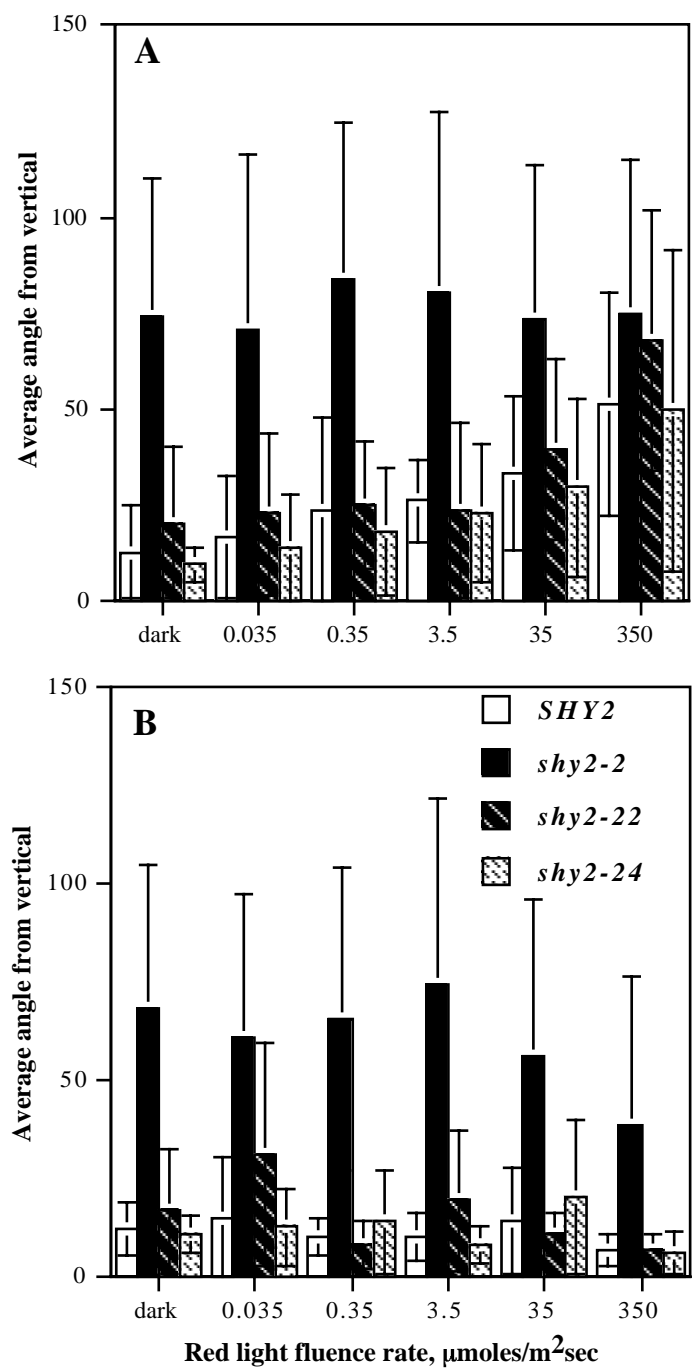

Fig. 6. Growth orientation with respect to gravity of wild-type and shy2 seedlings grown under different fluence rates of red light. (A) Hypocotyl, (B) root. Seedlings were grown on vertically oriented plates in red light for 5 days. The average angle of hypocotyl or root growth relative to gravity for each genotype is shown. Error bars indicate standard deviations. 25 seedlings were examined for each genotype. 

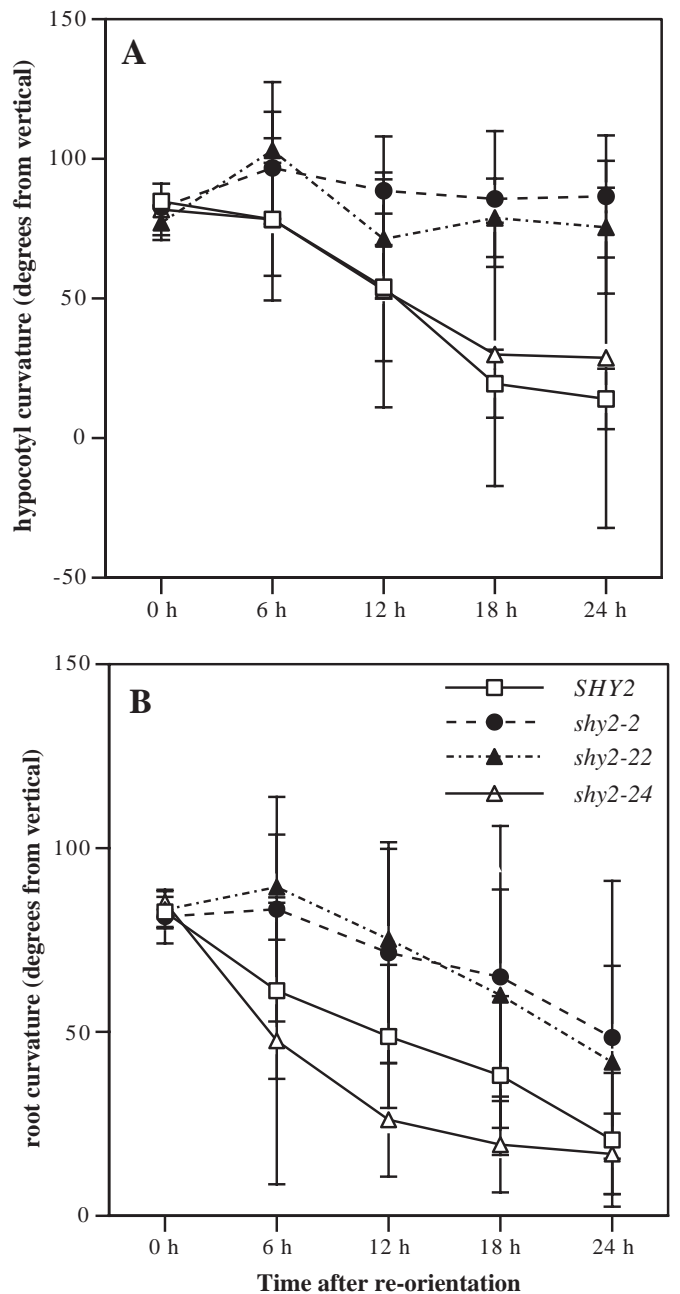

Fig. 7. Time course of gravitropic response of wild-type and shy2 seedlings. (A) Hypocotyl, (B) root. Dark grown seedlings were rotated by $90^{\circ}$ in darkness after 2 days growth. Curvature was measured every 6 hours after re-orientation. Error bars represent standard deviations. At least 20 seedlings were examined for each point.

tissues. However, this model would not explain simply why both gain-of-function and loss-of-function mutants have short roots.

A second possibility is that SHY2/IAA3 may act differently depending on its level in the cell. For example, weak or transient auxin signalling may induce a low level of SHY2/IAA3, sufficient to promote root growth or cotyledon expansion, but stronger or more persistent auxin signalling may induce a higher SHY2/IAA3 level, inhibiting these responses. In other systems, different concentrations of morphogens or transcription factors can either induce or repress particular genes (Ferguson and Anderson, 1992; Kosman et al., 1991). Consistent with this type of model, many auxin responses are biphasic, and auxin has been proposed to act as a morphogen in tissue patterning (Sachs, 1991; Uggla et al., 1998). Biphasic cellular responses to auxin may depend on the architecture of intracellular feedback control loops, for example, if above a threshold level SHY2/IAA3 represses its own gene expression or that of other auxin-induced genes. Such feedback loops
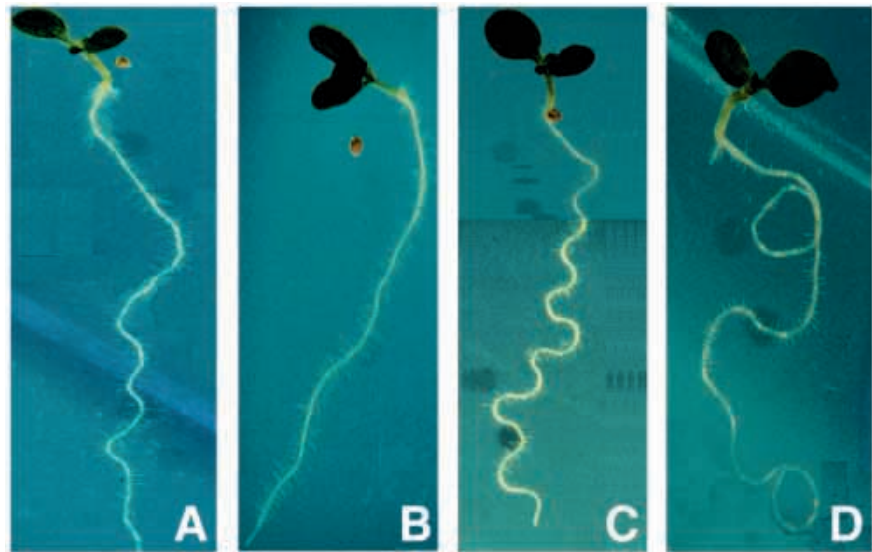

Fig. 8. Wavy roots of wild type and shy2 mutants. (A) Wild type, (B) shy2-2, (C) shy2-22, (D) shy2-24. Seedlings were grown on vertically oriented hard (1.5\% phytagar) plates in white light for 3 days, then the plates were tilted to $45^{\circ}$ and the seedlings photographed after another 3 days growth.

could also operate over time to give different responses in cells with different histories of auxin perception.

Previous biochemical and molecular studies provide data consistent with models that invoke positive or negative feedback control by SHY2/IAA3. Among the Arabidopsis $A u x / I A A$ genes characterized in detail by Abel et al. (IAAIIAA14), SHY2/IAA3 mRNA shows the most transient response to exogenous auxin. Induction begins after 5 minutes of treatment with IAA and reaches 4 -fold within 10 minutes. The mRNA level then decreases starting at 30 minutes and reaches starting mRNA levels after 4 hours (Abel et al., 1995). These results suggest that $S H Y 2 / I A A 3$ induction is a very early step in auxin responses, and that later steps re-establish repression of the SHY2/IAA3 gene. Since Aux/IAA proteins and ARF proteins can heterodimerize (Kim et al., 1997; Ulmasov et al., 1997b), SHY2/IAA3 may dimerize with particular ARFs to regulate expression of $A u x / I A A$ genes. In mediating auxin responses, SHY2/IAA3 might turn on other genes, and/or it might turn off genes including itself, thereby forming either positive or negative feedback loops.

A third model is that shy2 mutations affect auxin transport, and the mutant phenotypes arise secondarily from aberrant distribution of auxin. For example, gain-of-function shy2 mutants may have higher levels of auxin in cotyledons, resulting in excess expansion. In the shy2-24 putative null mutant, loss of SHY2/IAA3 protein may result in increased auxin transport from cotyledons and/or leaves to hypocotyl and root, resulting in a slightly longer hypocotyl, and more lateral roots than wild type. Similarly, auxin distribution within the root tissues may be altered, resulting in increased auxin in elongating cells and pericycle cells (causing faster gravitropism and more lateral root formation) and reduced auxin in the meristem (causing decreased growth and altered response to exogenous auxin). Consistent with this model, hypocotyl elongation in light-grown seedlings requires auxin transport (Jensen et al., 1998), and mutants that are defective in auxin transport have agravitropic root growth (Luschnig et al., 1998; Pickett et al., 1990).

Gravitropism of both roots and shoots arises from 
differential growth on opposite sides of the root or stem, and is thought to result from stimulation and/or inhibition of cell elongation by asymmetrically localized auxin (Goldsmith, 1977; Masson, 1995; Pickard, 1985; Thimann, 1977; Young et al., 1990). The altered waviness of shy2 loss-of-function mutant root growth may result from faster curvature of a gravityresponding root, or from decreased correction on the return wave, resulting in more persistent curving. The former model is consistent with the faster gravitropic response of dark-grown shy2-24 roots. In either case, the wavy root phenotype reveals that correct timing of auxin responses is important for normal growth. Depending on whether the curvature is caused by excess elongation on the outside of the curving root or too little elongation on the inside, the data may indicate a negative or positive effect of SHY2/IAA3 on elongation. More detailed study of this response may clarify this issue.

These studies of SHY2/IAA3 raise the question of what the other 19 Arabidopsis Aux/IAA proteins do, and how their activities may be related to those of SHY2/IAA3. The subtle shoot phenotypes of loss-offunction shy2 mutants suggest that SHY2/IAA3 may act redundantly in shoots with other proteins, possibly encoded by other $A u x / I A A$ genes. Perhaps $A X R 3 / I A A 17$ is one such gene, as axr3 gain-of-function mutants are similar to shy2-2 in having short hypocotyls, curled-up leaves, and leaf formation in the dark (Leyser et al., 1996). However, shy2 and axr3 mutant root phenotypes contrast in several ways. For example, gain-of-function axr3 mutants have more adventitious roots than wild type, whereas gainof-function shy2 mutants have fewer adventitious roots than wild type. These results suggest that their functions may diverge in controlling root growth. It is also possible that one of these genes regulates the other and the regulation is positive in shoots but negative in roots. Further genetic and molecular work may provide more information on the regulatory interactions among SHY2/IAA3, AXR3/IAA17, and other $A u x / I A A$ genes.

The most prominent phenotypes of loss-of-function shy 2
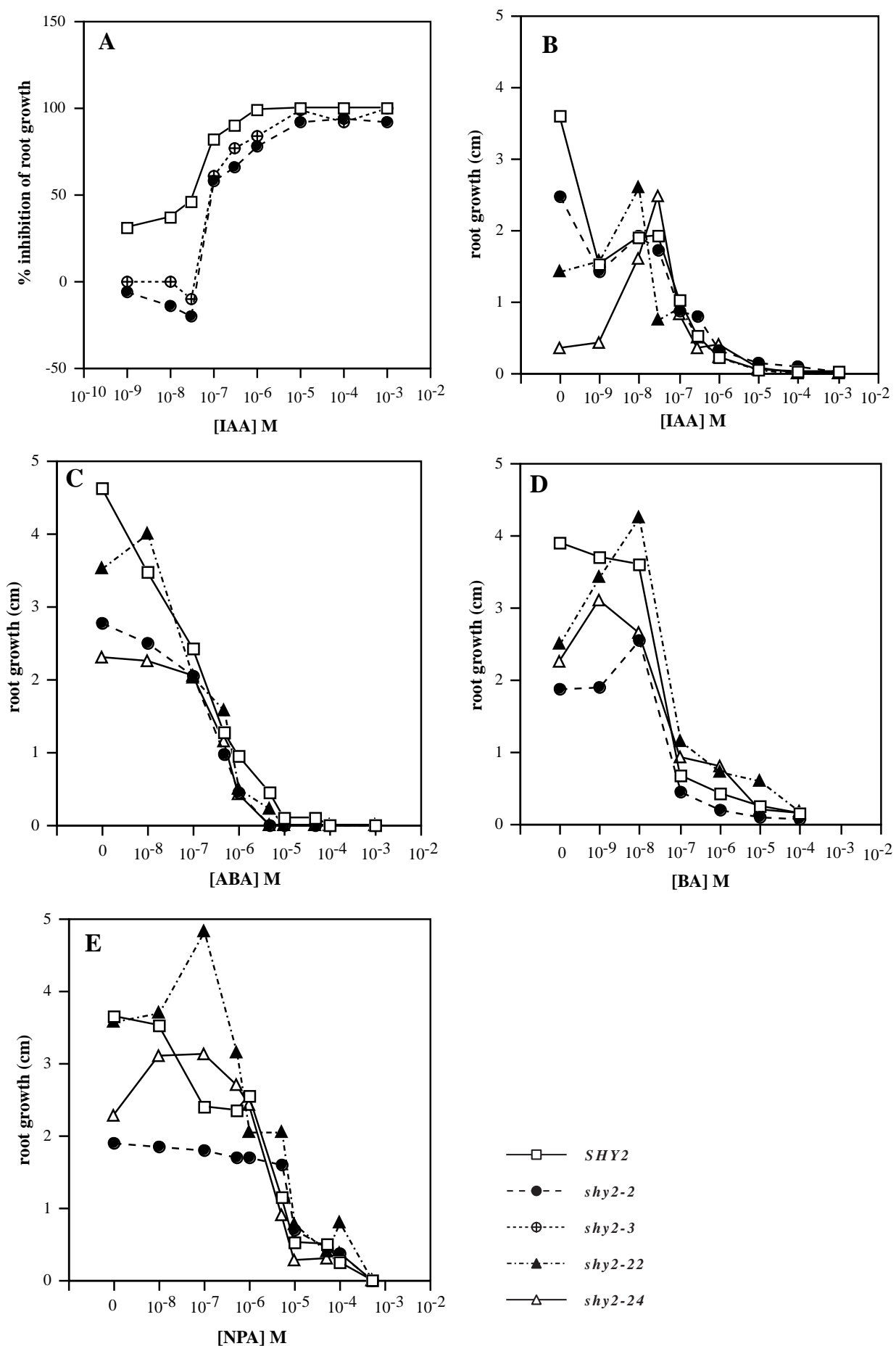

Fig. 9. Root growth in the presence of exogenous hormones. Dose-response curves for root growth on IAA (A,B), ABA (C), BA (D), and NPA (E). In A, inhibition of root growth is expressed relative to growth without hormone. B-E show root growth measurements. Error bars are omitted for clarity. Each point represents the mean of 20 measurements, and standard deviations were generally less than $20 \%$ of the mean. mutants occur in light-grown seedlings, suggesting that SHY2/IAA3 may be more important in light than in the dark. This is consistent with suggestions that auxin plays different roles in light- and dark-grown seedlings (Jensen et al., 1998). Moreover, the finding that shy2 gain-of-function mutants make leaves in the dark suggests that SHY2/IAA3 is normally part 
of the morphological program of light-grown plants, and that activation of SHY2/IAA3 by mutation may be sufficient to trigger de-etiolation (Kim et al., 1996, 1998; Reed et al., 1998). In addition to helping unravel auxin regulatory networks, shy 2 mutants will provide useful tools for detailed study of whether SHY2/IAA3 forms a functional link between light and morphological responses.

We thank Alan Jones for comments on the manuscript, Susan Whitfield for help with figures, and Ottoline Leyser for information on CAPS markers and axr3 mutations. This work was funded by National Institutes of Health grant R29-52456 to J. W. R.

\section{REFERENCES}

Abel, S., Nguyen, M. D. and Theologis, A. (1995). The PS-IAA4/5-like family of early auxin-inducible mRNAs in Arabidopsis thaliana. J. Mol. Biol. 251, 533-549.

Abel, S., Oeller, P. W. and Theologis, A. (1994). Early auxin-induced genes encode short-lived nuclear proteins. Proc. Natl. Acad. Sci. USA 91, 326-330.

Abel, S. and Theologis, A. (1995). A polymorphic bipartite motif signals nuclear targeting of early auxin-inducible proteins related to PS-IAA4 from pea (Pisum sativum). Plant J. 8, 87-96.

Abel, S. and Theologis, A. (1996). Early genes and auxin action. Plant Physiol. 111, 9-17.

Bechtold, N., Ellis, J. and Pelletier, G. (1993). In planta Agrobacterium mediated gene transfer by infiltration of adult Arabidopsis thaliana plants. C. R. Acad. Sci. Paris Life Sci. 316, 1194-1199.

Bennett, M. J., Marchant, A., Green, H. G., May, S. T., Ward, S. P., Millner, P. A., Walker, A. R., Schulz, B. and Feldmann, K. A. (1996). Arabidopsis AUX1 gene: a permease-like regulator of root gravitropism. Science 273, 948-950.

Bennett, S. R. M., Alvarez, J., Bossinger, G. and Smyth, D. R. (1995). Morphogenesis in pinoid mutants of Arabidopsis thaliana. Plant J. 8, 505520.

Carland, F. M. and McHale, N. A. (1996). LOP1: a gene involved in auxin transport and vascular patterning in Arabidopsis. Development 122, 18111819.

Cernac, A., Lincoln, C., Lammer, D. and Estelle, M. (1997). The SAR1 gene of Arabidopsis acts downstream of the AXR1 auxin response. Development 124, 1583-1591.

Chory, J., Nagpal, P. and Peto, C. A. (1991). Phenotypic and genetic analysis of $\operatorname{det} 2$, a new mutant that affects light-regulated seedling development in Arabidopsis. Plant Cell 3, 445-459.

del Pozo, J. C., Timpte, C., Tan, S., Callis, J. and Estelle, M. (1998). The ubiquitin-related protein RUB1 and auxin responses in Arabidopsis. Science 280, 1760-1763.

Estelle, M. and Klee, H. J. (1994) Auxin and cytokinin in Arabidopsis. In Arabidopsis (ed. E. M. Meyerowitz and C.R. Somerville), pp 555-578. Cold Spring Harbor, NY: Cold Spring Harbor Laboratory Press.

Evans, M. L., Ishikawa, H. and Estelle, M. A. (1994). Responses of Arabidopsis roots to auxin studied with high temporal resolution: Comparison of wild type and auxin-response mutants. Planta 194, 215-222.

Ferguson, E. L. and Anderson, K. V. (1992). decapentaplegic acts as a morphogen to organize dorsal-ventral pattern in the Drosophila embryo. Cell 71, 451-461.

Fujita, H. and Syono, K. (1997). PIS1, a negative regulator of the action of auxin transport inhibitors in Arabidopsis thaliana. Plant J. 12, 583-595.

Gaiser, J. C. and Lomax, T. L. (1993). The altered gravitropic response of the lazy-2 mutant of tomato is phytochrome-related. Plant Physiol. 102, 339344.

Garbers, C., DeLong, A., Deruére, J., Bernasconi, P. and Söll, D. (1996). A mutation in protein phosphatase $2 \mathrm{~A}$ regulatory subunit A affects auxin transport in Arabidopsis. EMBO J. 15, 2115-2124.

Goldsmith, M. H. M. (1977). The polar transport of auxin. Ann. Rev. Plant Physiol. 28, 439-478.

Guilfoyle, T., Hagen, G., Ulmasov, T. and Murfett, J. (1998a). How does auxin turn on genes? Plant Physiol. 118, 341-347.

Guilfoyle, T. J., Ulmasov, T. and Hagen, G. (1998b). The ARF family of transcription factors and their role in plant hormone-responsive transcription. Cell Mol. Life Sci. 54, 619-627.

Hajdukiewicz, P., Svab, Z. and Maliga, P. (1994). The small, versatile $p P Z P$ family of Agrobacterium binary vectors for plant transformation. Plant Mol. Biol. 25, 989-994.

Hardtke, C. S. and Berleth, T. (1998). The Arabidopsis gene MONOPTEROS encodes a transcription factor mediating embryo axis formation and vascular development. EMBO J. 17, 1405-1411.

Hobbie, L. and Estelle, M. (1995). The axr4 auxin-resistant mutants of Arabidopsis thaliana define a gene important for root gravitropism and lateral root initiation. Plant J. 7, 211-220.

Hobbie, L. J. (1998). Auxin: molecular genetic approaches in Arabidopsis Plant Physiol. Biochem. 36, 91-102.

Jensen, P. J., Hangarter, R. P. and Estelle, M. (1998). Auxin transport is required for hypocotyl elongation in light-grown but not dark-grown Arabidopsis. Plant Physiol. 116, 455-462.

Kim, B. C., Soh, M. S., Hong, S. H., Furuya, M. and Nam, H. G. (1998). Photomorphogenic development of the Arabidopsis shy2-1D mutation and its interaction with phytochromes in darkness. Plant $J \mathbf{1 5}, 61-68$.

Kim, B. C., Soh, M. S., Kang, B. J., Furuya, M. and Nam, H. G. (1996). Two dominant photomorphogenic mutations of Arabidopsis thaliana identified as suppressor mutations of hy2. Plant J. 9, 441-456.

Kim, J., Harter, K. and Theologis, A. (1997). Protein-protein interactions among the Aux/IAA proteins. Proc. Natl. Acad. Sci. USA 94, 11786-11791.

Koncz, C. and Schell, J. (1986). The promoter of the $T_{L}-D N A$ gene 5 controls the tissue specific expression of chimeric genes carried by a novel type of Agrobacterium binary vector. Mol. Gen. Genet. 204, 383-396.

Konieczny, A. and Ausubel, F. (1993). A procedure for mapping Arabidopsis mutations using co-dominant ecotype-specific PCR-based markers. Plant J. 4, 403-410.

Kosman, D., Ip, Y. T., Levine, M. and Arora, K. (1991). Establishment of the mesoderm-neuroectoderm boundary in the Drosophila embryo. Science 254, 118-122.

Leyser, H. M. O., Pickett, F. B., Dharmasiri, S. and Estelle, M. (1996). Mutations in the $A X R 3$ gene of Arabidopsis result in altered auxin response including ectopic expression from the SAUR-ACl promoter. Plant J. 10, 403-413.

Liscum, E. and Hangarter, R. P. (1993). Genetic evidence that the Pr form of phytochrome B mediates gravitropism in Arabidopsis thaliana. Plant Physiol. 103, 15-19.

Luschnig, C., Gaxiola, R. A., Grisafi, P. and Fink, G. R. (1998). EIR1, a root-specific protein involved in auxin transport, is required for gravitropism in Arabidopsis thaliana. Genes Dev. 12, 2175-2187.

Masson, P. H. (1995). Root gravitropism. BioEssays 17, 119-127.

Okada, K. and Shimura, Y. (1990). Reversible root tip rotation in Arabidopsis seedlings induced by obstacle-touching stimulus. Science $\mathbf{2 5 0}$, 274-276.

Okada, K. and Shimura, Y. (1994) Modulation of root growth by physical stimuli. Arabidopsis (ed. E. M. Meyerowitz and C. R. Somerville), pp. 665684. Cold Spring Harbor, NY: Cold Spring Harbor Laboratory Press.

Okada, K., Ueda, J., Komaki, M. K., Bell, C. J. and Shimura, Y. (1991). Requirement of the auxin polar transport system in early stages of Arabidopsis floral bud formation. Plant Cell 3, 677-684.

Oyama, T., Shimura, Y. and Okada, K. (1997). The Arabidopsis HY5 gene encodes a bZIP protein that regulates stimulus-induced development of root and hypocotyl. Genes Dev. 11, 2983-2995.

Pickard, B. G. (1985). Early events in geotropism of seedling shoots. Ann. Rev. Plant Physiol. 36, 55-75.

Pickett, F. B., Wilson, A. K. and Estelle, M. (1990). The auxl mutation of Arabisopsis confers both auxin and ethylene resistance. Plant Physiol. 94 1462-1466.

Przemeck, G. K. H., Mattsson, J., Hardtke, C. S., Sung, Z. R. and Berleth, T. (1996). Studies on the role of the Arabidopsis gene MONOPTEROS in vascular development and plant cell axialization. Planta 200, 229-237.

Reed, J. W., Elumalai, R. P. and Chory, J. (1998). Suppressors of an Arabidopsis thaliana phyB mutation identify genes that control light signalling and hypocotyl elongation. Genetics 148, 1295-1310.

Robson, P. R. H. and Smith, H. (1996). Genetic and transgenic evidence that phytochromes A and B act to modulate the gravitropic orientation of Arabidopsis thaliana hypocotyls. Plant Physiol. 110, 211-216.

Rouse, D., Mackay, P., Stirnberg, P., Estelle, M. and Leyser, O. (1998). Changes in auxin response from mutations in an AUX/IAA gene. Science 279, 1371-1373.

Ruegger, M., Dewey, E., Gray, W. M., Hobbie, L., Turner, J. and Estelle, 
M. (1998). The TIR1 protein of Arabidopsis functions in auxin response and is related to human SKP2 and yeast Grr1p. Genes Dev. 12, 198-207.

Ruegger, M., Dewey, E., Hobbie, L., Brown, D., Bernasconi, P., Turner, J., Muday, G. and Estelle, M. (1997). Reduced naphthylphthalamic acid binding in the tir3 mutant of Arabidopsis is associated with a reduction in polar auxin transport and diverse morphological defects. Plant Cell 9, 745-757.

Sachs, T. (1991) Pattern Formation in Plant Tissues. Cambridge University Press, Cambridge.

Sessions, A., Nemhauser, J. L., McColl, A., Roe, J. L., Feldmann, K. A. and Zambryski, P. C. (1997). ETTIN patterns the Arabidopsis floral meristem and reproductive organs. Development 124, 4481-4491.

Simmons, C., Söll, D. and Migliaccio, F. (1995). Circumnutation and gravitropism cause root waving in Arabidopsis thaliana. J. Exp. Bot. 46, 143-150.

Thimann, K. V. (1977) Hormone action in the whole life of plants. University of Massachusetts Press, Amherst.

Uggla, C., Mellerowicz, E. J. and Sundberg, B. (1998). Indole-3-acetic acid controls cambial growth in Scots pine by positional signaling. Plant Physiol. 117, 113-121.

Ulmasov, T., Hagen, G. and Guilfoyle, T. J. (1997a). ARF1, a transcription factor that binds to auxin response elements. Science 276, 1865-1868.

Ulmasov, T., Murfett, J., Hagen, G. and Guilfoyle, T. J. (1997b). Aux/IAA proteins repress expression of reporter genes containing natural and highly active synthetic auxin response elements. Plant Cell 9, 1963-1971.

Wilson, A. K., Pickett, F. B., Turner, J. C. and Estelle, M. (1990). A dominant mutation in Arabidopsis confers resistance to auxin, ethylene and abscisic acid. Mol. Gen. Genet. 222, 377-383.

Yamamoto, M. and Yamamoto, K. T. (1998). Differential effects of 1naphthyleneacetic acid, indole-3-acetic acid and 2,4-dichlorophenoxyacetic acid on the gravitropic response of roots in an auxin-resistant mutant of Arabidopsis, aux1. Plant Cell Physiol. 39, 660-664.

Young, L. M., Evans, M. L. and Hertel, R. (1990). Correlations between gravitropic curvature and auxin mvement across gravistimulated roots of Zea mays. Plant Physiol. 92, 792-796. 